\title{
Prognostic implications of human papillomavirus type 16 status in non-oropharyngeal head and neck cancer: a propensity score matching analysis
}

\author{
Yingying Zhu ${ }^{1}$, Xin $\mathrm{Xia}^{1}$, Liming Gao ${ }^{1}$, Xiaoli Zhu ${ }^{1}$, Wenwen Diao ${ }^{1}$, Zhiyong Liang ${ }^{2}$, Zhiqiang Gao ${ }^{1}$, \\ Xingming Chen ${ }^{1}$ \\ ${ }^{1}$ Department of Otolaryngology-Head and Neck Surgery, China; ${ }^{2}$ Department of Pathology, Peking Union Medical College Hospital, Peking Union \\ Medical College and Chinese Academy of Medical Sciences, Beijing 100730, China \\ Contributions: (I) Conception and design: Y Zhu, X Chen; (II) Administrative support: Z Gao; (III) Provision of study materials or patients: X Zhu, \\ W Diao, Z Liang, X Chen; (IV) Collection and assembly of data: Y Zhu, X Xia; (V) Data analysis and interpretation: Y Zhu, X Xia, X Chen; (VI) \\ Manuscript writing: All authors; (VII) Final approval of manuscript: All authors. \\ Correspondence to: Xingming Chen, MD. Department of Otolaryngology-Head and Neck Surgery, Peking Union Medical College Hospital, \\ Peking Union Medical College and Chinese Academy of Medical Sciences, No. 1, Shuaifuyuan, Wangfujing, Beijing 100730, China. \\ Email: xingming.chen@hotmail.com.
}

\begin{abstract}
Background: The purpose of this study was to investigate the association between HPV 16 status and survival outcomes in patients with non-oropharyngeal squamous carcinoma (non-OPSCC).

Methods: Patients with non-OPSCC diagnosed between 2006 and 2016 were included in this study. The presence of HPV 16 DNA was confirmed by quantitative real-time polymerase chain reaction. Survival analysis was performed using Kaplan-Meier estimates, the Cox proportional hazards model, and propensity score matching (PSM).

Results: Overall, 1,539 patients with adequate specimens were identified, of whom $131(8.51 \%)$ were positive for HPV 16. Compared to HPV 16-negative patients, the adjusted hazard ratios (aHR) for HPV 16-positive non-OPSCC patients were 0.77 and 0.81 for disease-specific survival (DSS) and overall survival (OS), respectively. Additionally, the larynx was the only subsite in which DSS was significantly improved. After PSM, cohorts consisted of 129 patients in the HPV 16-positive group and 129 in the HPV 16-negative group. HPV 16-positive non-OPSCC patients had favorable survival outcomes, however, these outcomes were not significantly different compared to HPV 16-negative patients. Stratified analysis performed according to primary site showed that only in the larynx was HPV 16-positive status a significant factor for predicting favorable DSS.
\end{abstract}

Conclusions: Our findings indicate that HPV 16-positive non-OPSCC patients did not have significantly better survival outcomes compared to HPV 16-negative patients.

Keywords: Human papillomavirus; non-oropharyngeal neoplasms; head and neck cancers; squamous cell carcinoma; prognosis

Submitted Jul 11, 2019. Accepted for publication Nov 05, 2019.

doi: 10.21037/atm.2019.11.72

View this article at: http://dx.doi.org/10.21037/atm.2019.11.72

\section{Introduction}

Globally, head and neck cancers accounted for an estimated 705,781 malignancies and 358,108 deaths in 2018 (1); combined, they are the sixth most common type of cancer worldwide, more than $90 \%$ of which is squamous cell carcinoma (2). Head and neck squamous cell carcinoma (HNSCC) is a heterogeneous disease occurring in various sites within the head and neck region, including the larynx, 
oropharynx, oral cavity, and hypopharynx. The most common risk factors are tobacco and alcohol use and highrisk human papillomavirus (HPV) infection $(3,4)$. Although detection rates vary based on detection method and study populations, approximately $24.9 \%$ to $73.1 \%$ of patients with oropharyngeal squamous cell carcinomas (OPSCCs) and $6.1 \%$ to $28.8 \%$ of patients with non-oropharyngeal head and neck squamous cell carcinomas (non-OPSCCs), which include cancers of the larynx, oral cavity, and hypopharynx, are HPV-positive (5-10). Although several types of HPV have carcinogenic potential, the HPV 16 subtype accounts for more than $90 \%$ of HPV-positive HNSCCs $(6,7,11,12)$.

Previous studies have shown that patients with HPVpositive OPSCC are clinically distinct from HPV-negative patients and have a better prognosis $(7,13)$. However, the underlying role of HPV 16 in squamous cell carcinomas of the head and neck in regions outside the oropharynx and its relationship to clinical outcomes has not been established (14-16).

The aim of this study was to investigate the prevalence of HPV 16 and its association with survival outcomes in a large non-OPSCC patient cohort. Both Cox proportional hazard regression analysis and propensity scoring methods were used to reduce the effect of confounding variables.

\section{Methods}

\section{Study participants}

The Peking Union Medical College Hospital (PUMCH) Ethics Committee approved this study protocol. All participants provided informed written consent. Previously untreated non-OPSCC patients who were examined at PUMCH between January 2006 and January 2016 were enrolled in this study. Diagnosis was based on specimens obtained from surgery or biopsy, and each was reviewed by an experienced pathologist. Exclusion criteria included second primary tumor; primary tumor site other than the oral cavity, larynx, or hypopharynx; histopathologic diagnosis other than squamous cell carcinoma; distant metastasis; previous palliative treatment; inadequate specimens; and inadequate follow-up data. A flowchart describing subject recruitment and exclusion is shown in Figure 1 .

Clinical information was extracted from patient medical records and included gender, age, tobacco and alcohol consumption, medical comorbidity score, histopathologic grade, tumor site, stage, and primary treatment modality. Smokers were defined as patients who had smoked more than 100 cigarettes in their lifetime, whereas nonsmokers had smoked 100 cigarettes or fewer. Drinkers were defined as patients who drank alcoholic beverages at least once a week for more than 1 year during their lifetime; patients who did not meet this criterion were defined as nondrinkers. Medical comorbidity scores were classified according to the Adult Comorbidity Evaluation-27 (ACE-27) (17). All patients were treated with curative intent, and HPV status was unknown at the time of treatment. Treatment decisions were based on the existing policies of the PUMCH Head and Neck Tumor Multidisciplinary Team and were individualized according to patient age, tumor stage, primary subsite, and medical comorbidities. The surgical treatment group included patients receiving surgery only, surgery plus radiotherapy, or surgery plus chemoradiotherapy. The nonsurgical treatment group included patients receiving definitive radiotherapy and/or chemoradiotherapy.

\section{Patient follow-up and study end points}

All patients received clinical and radiographic examinations every 3 months in the first to third year, every 6 months in the fourth and fifth year, and annually thereafter. Patients were recorded as living and free of recurrence if disease-free status was confirmed on the date of the last visit. All patients were followed-up for a minimum of 36 months, or until an outcome of interest occurred.

The primary end point was overall survival (OS) and the secondary end point was disease-specific survival (DSS). OS was defined as the time elapsed between initiation of treatment and death from any cause. DSS was defined as the time between the initiation of treatment and death from disease.

\section{DNA extraction}

Tumor specimens were microdissected to ensure that only samples containing more than $70 \%$ malignant epithelial cells were analyzed. DNA was extracted and purified from fresh frozen and formalin-fixed paraffin-embedded (FFPE) samples using a QIAamp DNA Mini Kit (Qiagen, Valencia, CA, USA) and a QIAamp DNA FFPE Tissue Kit (Qiagen Ltd., Hilden, Germany), respectively. PCR inhibition was monitored by amplification of a 268-bp fragment of the human $\beta$-globin gene (GenBank accession: L09267.1) using $\mathrm{PCO} 4$ and $\mathrm{GH} 2 \mathrm{O}$ primers (18). Extracted DNA was stored at $-20^{\circ} \mathrm{C}$ prior to PCR analysis. 


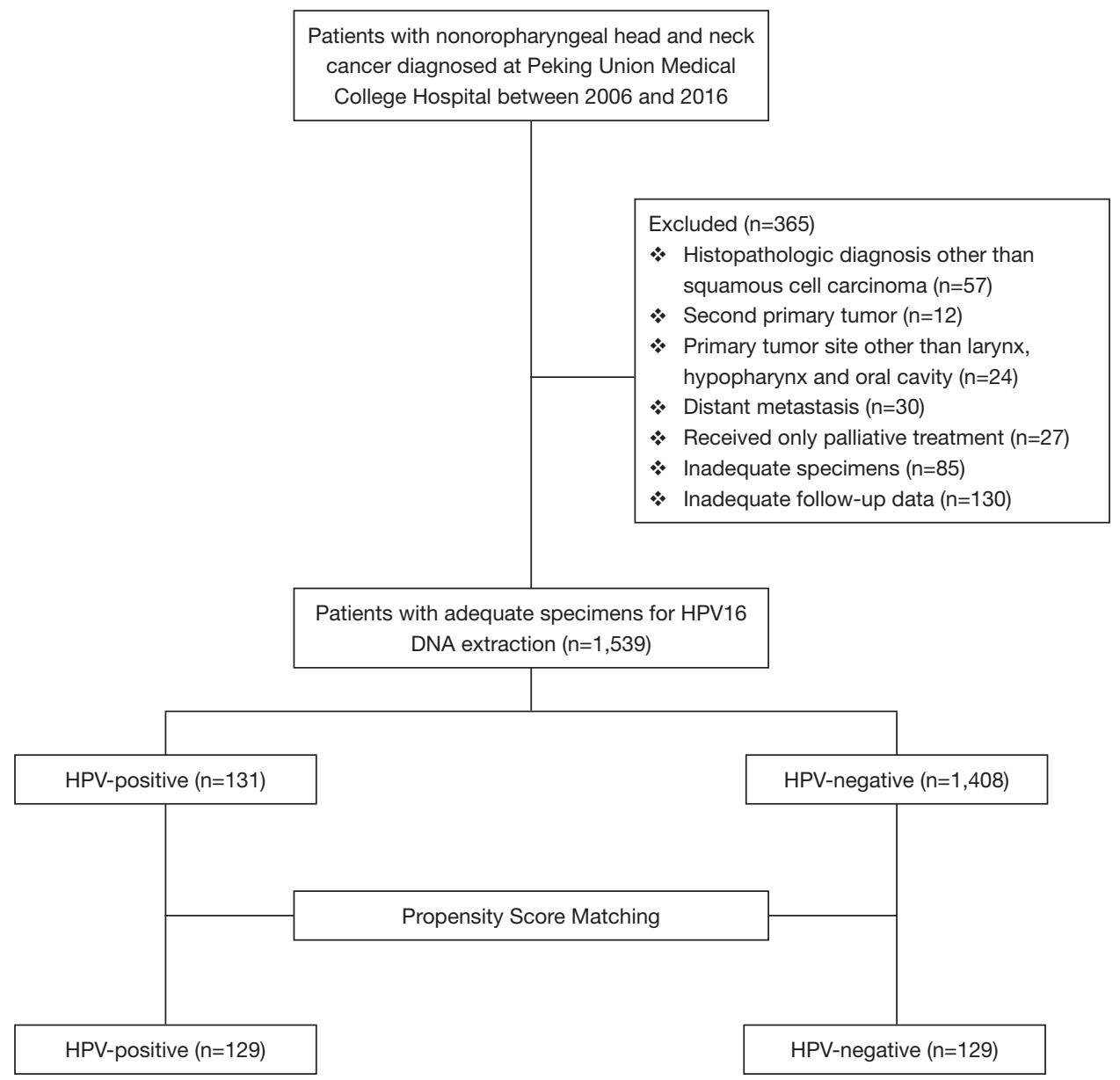

Figure 1 Flowchart describing patient enrollment and propensity score matching.

\section{HPV 16 DNA detection}

HPV 16 DNA was detected using quantitative real-time PCR (qRT-PCR) with Rotor Gene 6000 and SYBR Premix Ex Taq (Perfect Real Time) (TaKaRa DRR063A). Primers and FAM-MGB-labeled TaqMan probes were designed and optimized to specifically amplify the HPV 16 E6 region. The HPV 16-positive cervical cancer cell lines CaSki and $\mathrm{SiHa}$ (ATCC-LGC-HTB-35) were used as positive controls and calibrators for the assay. The detection threshold for HPV-positive status was set, and samples were deemed positive if the threshold was met in duplicate runs.

\section{Propensity score matching (PSM)}

PSM analysis is a superior and more refined statistical method of adjusting for potential baseline confounding variables. To reduce bias from confounding factors between groups, PSM was performed with a tolerance of 0.05 . Matching covariates consisted of gender, age, tobacco and alcohol consumption, adult comorbidity score, histopathologic grade, tumor site, stage, and primary treatment modality.

\section{Statistical analysis}

Categorical data were reported as absolute numbers (n) with proportion (\%). Groups were compared using Pearson's $\chi^{2}$ test. Survival analysis was carried out using the KaplanMeier method and was compared to the log-rank test for equality of survival curves. Univariate analyses were performed with the unadjusted Cox proportional hazards model to calculate the hazard ratio (HR). Multivariate analyses were also performed using the Cox proportional hazards model to identify independent prognostic factors 
through backward elimination. PSM was carried out as described above. All statistical tests were two-tailed, and $\mathrm{P}$ values less than 0.05 were considered significant. Data were analyzed using SPSS (Windows version 22.0; Chicago, IL, USA).

\section{Results}

\section{Patient characteristics}

A total of 1,539 patients had complete clinical data and adequate specimens. Overall, 131 (8.51\%) patients were positive for HPV 16 DNA (9.49\% of larynx cases, $6.81 \%$ of hypopharynx cases, and $8.03 \%$ of oral cavity cases). HPV 16 was present more often in females $(15.9 \%$ of females vs. $8.1 \%$ of males, $\mathrm{P}=0.014)$, nonsmokers $(11.0 \%$ of nonsmokers vs. $7.6 \%$ of smokers, $\mathrm{P}=0.036$ ), patients with poorly differentiated tumors (11.0\% of poorly differentiated tumors $v s .9 .6 \%$ of moderately differentiated tumors $v s$. $6.7 \%$ of well differentiated tumors, $\mathrm{P}=0.047$ ), and patients who received nonsurgical treatment $(11.3 \%$ of nonsurgical treatment patients vs. $7.5 \%$ of surgical treatment patients, $\mathrm{P}=0.016$ ). Baseline patient characteristics are summarized in Table 1.

\section{Survival outcomes}

Survival outcomes were measured and compared based on HPV 16 status. The median follow-up time was 48 months (range, 8-154 months). According to the Kaplan-Meier method, there was no significant difference in OS (3-year: $77 \%$ vs. $69.8 \%, 5$-year: $48.2 \%$ vs. $46.3 \%, \mathrm{P}=0.136)$ or DSS (3-year: $80 \%$ vs. $70.5 \%, 5$-year: $53.6 \%$ vs. $49 \%, \mathrm{P}=0.091$ ) between patients with HPV 16-positive and -negative tumors (Figure 2A).

Based on unadjusted and adjusted Cox analyses, HPV 16-positive status was associated with improved OS (unadjusted HR: 0.84; 95\% CI: 0.66-1.06, $\mathrm{P}=0.140$; adjusted HR: 0.81 ; 95\% CI: $0.64-1.03, \mathrm{P}=0.090)$ and DSS (unadjusted HR: 0.80; 95\% CI: 0.62-1.04, $\mathrm{P}=0.094$; adjusted HR: 0.77; 95\% CI: 0.60-1.00, P=0.051), although these results were not statistically significant (Table 2).

\section{Propensity matched analysis}

After PSM, 129 patients were included in the HPV 16-positive group and 129 were included in the HPV 16-negative group. Preexisting differences between groups were well balanced, as shown in Table 1. However, no survival benefits were observed between HPV 16-positive and -negative groups for 3- and 5-year OS (3-year: 76.0\% vs. $70.5 \%, 5$-year: $48.7 \%$ vs. $46.3 \%, \mathrm{P}=0.082)$ or DSS (3-year: $79.6 \%$ vs. $74.9 \%$, 5 -year: $54.6 \%$ vs. $49.9 \%$, $\mathrm{P}=0.123$ ) (Figure 2B).

In univariate and multivariate Cox analyses, patients with HPV 16-positive tumors had favorable outcomes compared to patients with HPV 16-negative tumors for both OS (unadjusted HR: 0.76; 95\% CI: 0.55-1.04, $\mathrm{P}=0.086$, adjusted HR: $0.74 ; 95 \% \mathrm{CI}: 0.53-1.02, \mathrm{P}=0.070)$ and DSS (unadjusted HR: 0.76; 95\% CI: 0.54-1.08, $\mathrm{P}=0.126$, adjusted HR: 0.72; 95\% CI: 0.50-1.02, $\mathrm{P}=0.065)$, although these results were not significant (Table 2).

\section{Survival outcomes of HPV16 DNA status stratified by subsite}

Survival outcomes were also compared based on primary subsite (larynx, hypopharynx, or oral cavity). Kaplan-Meier analysis revealed statistically significant improvement in survival outcomes for larynx non-OPSCC patients (Figure 3). Adjusted Cox analyses in cohort data revealed that HPV 16-positive larynx tumors were associated with improved OS and DSS compared to patients with HPV 16-negative tumors (Cohort: OS, adjusted HR: 0.70; 95\% CI: 0.47-1.03, P=0.070; DSS, adjusted HR: 0.60; 95\% CI: 0.39-0.93, $\mathrm{P}=0.022$; PSM: OS, adjusted HR: 0.59; $95 \%$ CI: 0.34-0.99, P=0.049; DSS, adjusted HR: 0.52; 95\% CI: $0.28-0.95, \mathrm{P}=0.034$ ) (Table 2). No significant differences were observed in OS and DSS for hypopharynx and oral cavity tumors (Figures 4,5).

\section{Discussion}

In this study, we investigated the association between HPV status and survival outcomes in a large cohort of patients with non-OPSCC. The prevalence of HPV 16 in this cohort was $8.51 \%$, which was comparable to previous studies. Some studies reported HPV detection rates of up to $30 \%$ (10). This variability may be due to the use of alternate detection methods or differences between study populations.

PSM has been increasingly used as a statistical tool in observational studies. Clinical and demographic patient characteristics can be balanced between groups, thus mimicking randomized controlled trial design (19). The present study is the first to use both Cox regression and 
Table 1 Baseline characteristics before and after propensity score matching

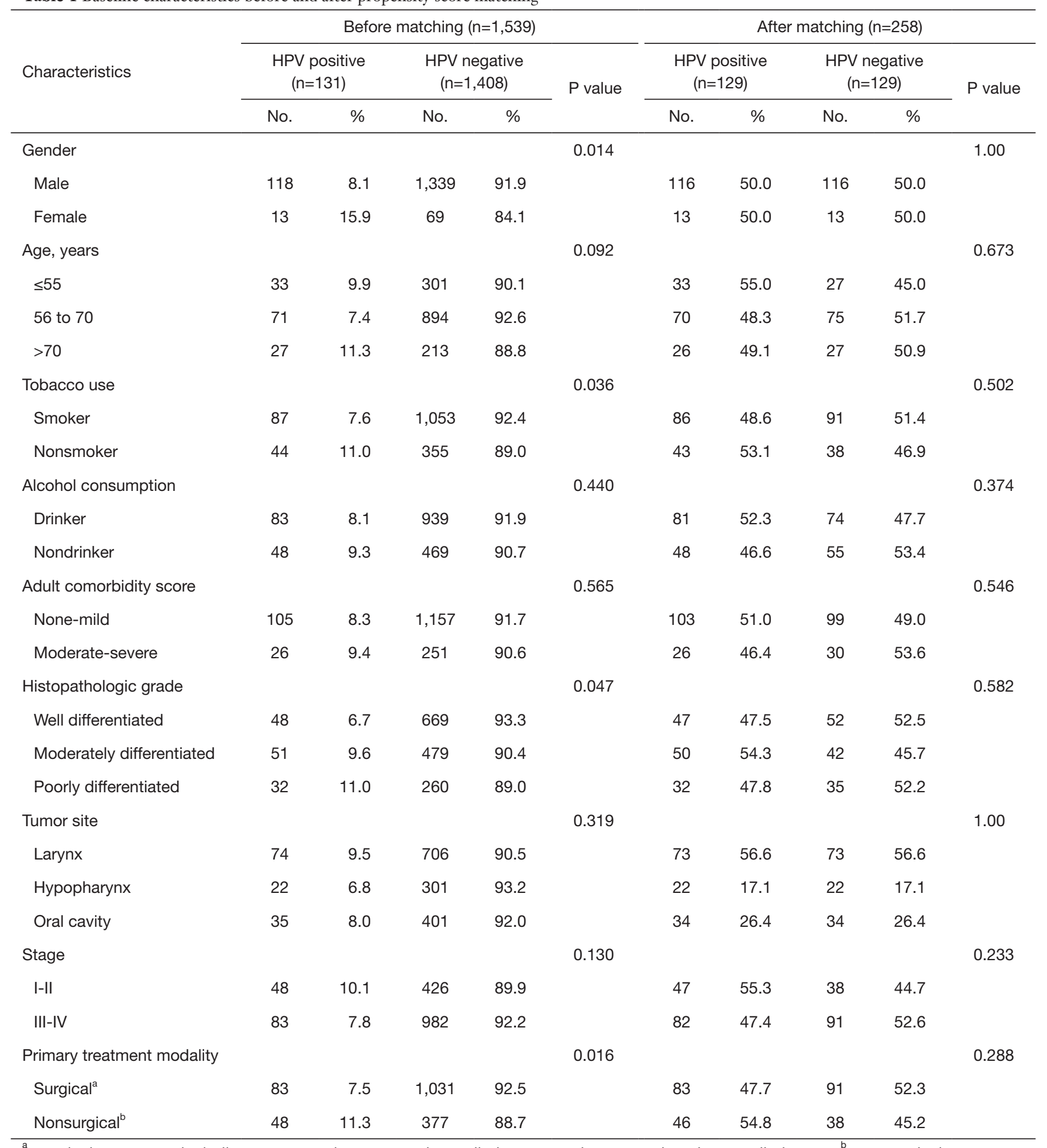

a , surgical treatment, including surgery only, surgery plus radiotherapy, and surgery plus chemoradiotherapy; ${ }^{\mathrm{b}}$, nonsurgical treatment, including definitive radiotherapy and chemoradiotherapy. HPV, human papillomavirus. 
A

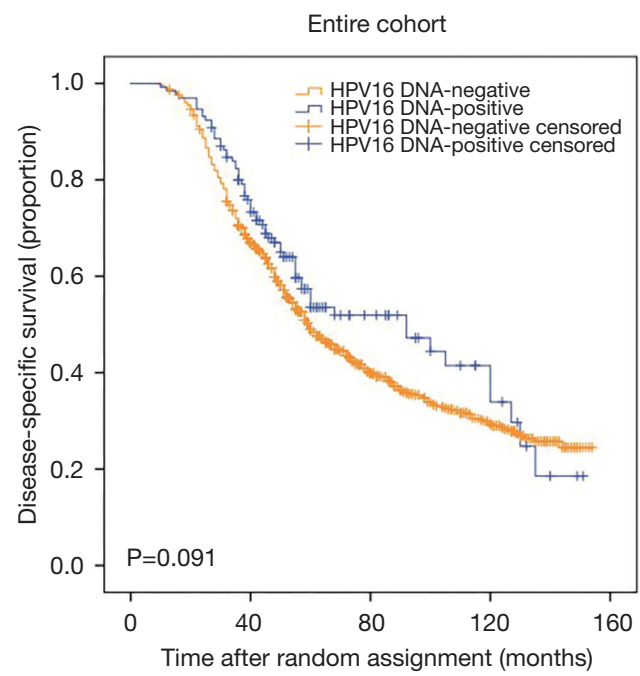

No. at risk

$\begin{array}{lccccc}\text { HPV-positive } & 131 & 86 & 26 & 9 & 0 \\ \text { HPV-negative } & 1,408 & 833 & 239 & 91 & 0\end{array}$

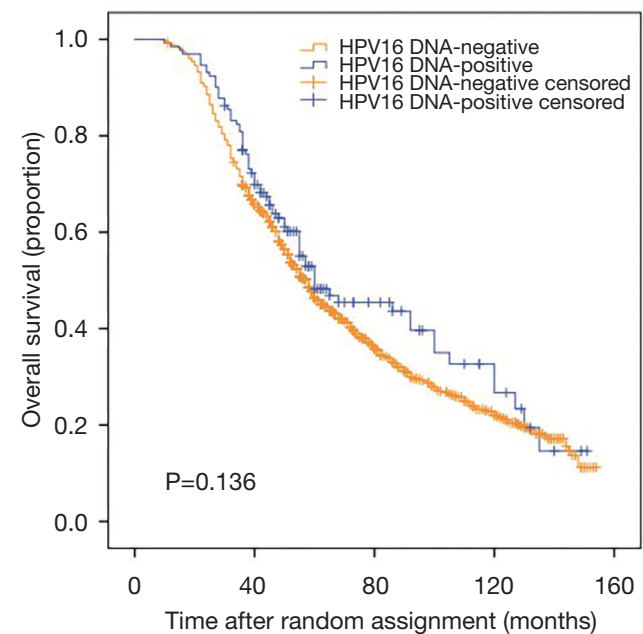

No. at risk

$\begin{array}{llllll}\text { HPV-positive } & 131 & 86 & 26 & 9 & 0\end{array}$

$\begin{array}{llllll}\text { HPV-negative } & 1,408 & 852 & 239 & 89 & 0\end{array}$
B

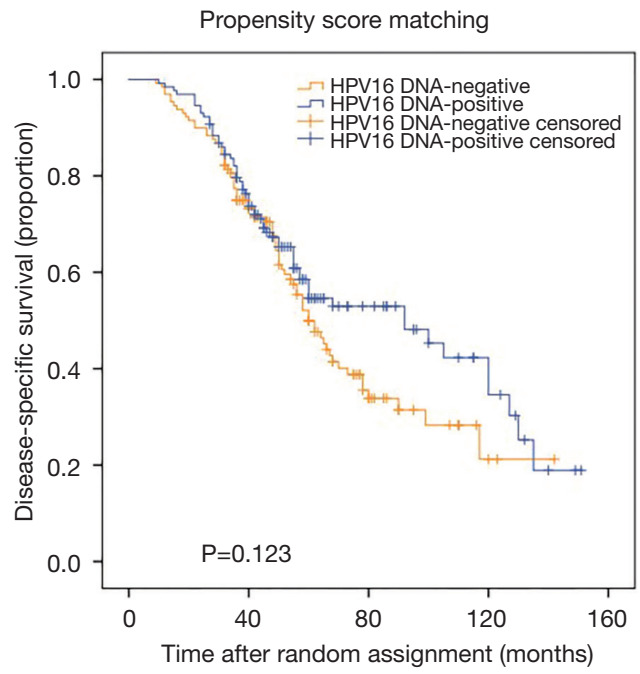

No. at risk

$\begin{array}{llllll}\text { HPV-positive } & 129 & 85 & 26 & 9 & 0 \\ \text { HPV-negative } & 129 & 81 & 18 & 2 & 0\end{array}$

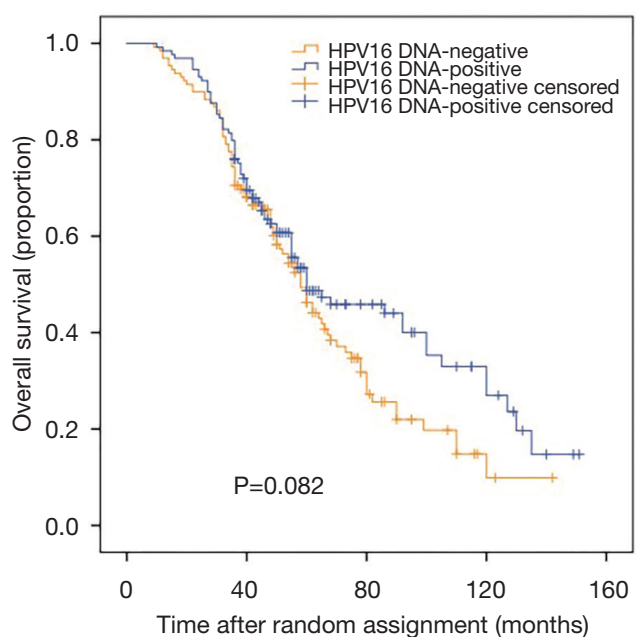

No. at risk $\begin{array}{llllll}\text { HPV-positive } & 129 & 85 & 26 & 9 & 0 \\ \text { HPV-negative } & 129 & 81 & 18 & 2 & 0\end{array}$

Figure 2 Disease-specific survival (DSS) and overall survival (OS) in HPV 16-positive and -negative non-OPSCC patients before (A) and after (B) propensity score matching.

propensity scoring methods to compare survival outcomes between HPV-positive and HPV-negative patients with non-OPSCC by adjusting for possible confounding factors. We chose to perform both PSM and adjusted Cox regression analysis due to limitations of both methods. Some authors have suggested that PSM outperforms standard multivariable methods (20). For our purposes, the advantages of one method did not outweigh the other; therefore, both methods were utilized.

In contrast to the strong prognostic role of HPV 16 status in OPSCC, here we show that patients with HPV 16-positive non-OPSCC had improved OS and DSS, but these improvements were not statistically significant. These findings are consistent with a similar study of 297 non-OPSCC cases, which showed that patients with $\mathrm{HPV}$-positive tumors did not have significantly better 
Table 2 Univariate and multivariate Cox regression analyses of HPV status with DSS and OS in the cohort study and after propensity score matching

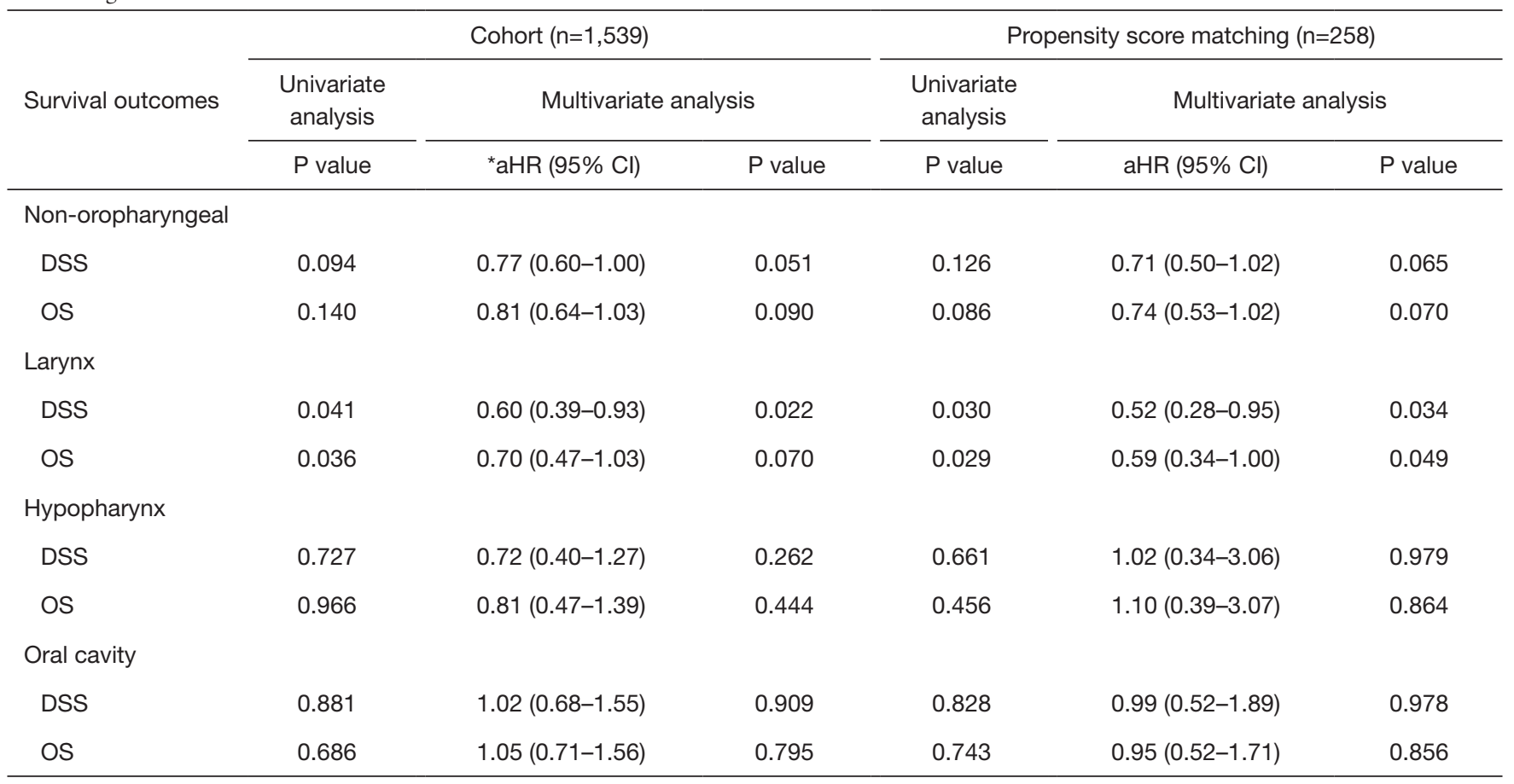

*, aHR estimated from Cox models stratified by protocol. aHR, adjusted hazard ratio; DSS, disease-specific survival; OS, overall survival.

progression-free survival (PFS) (HR: 0.77; 95\% CI: 0.44$1.33, \mathrm{P}=0.35$ ) or OS (HR: 0.64; 95\% CI: $0.34-1.21, \mathrm{P}=0.17$ ) compared to patients with HPV-negative tumors (15). Similarly, Fakhry et al., evaluated a retrospective, multiinstitutional cohort and found no significant association between HPV status and survival in non-OPSCC patients (HR: 1.18; 95\% CI: 0.69-2.01) (21). In contrast, a study by Nelson et al., showed that high-risk HPV status (indicated by seropositivity for HPV 16 E6 and E7) was associated with improved OS in oral cavity squamous cell carcinoma (OCSCC) (HR: 0.45; 95\% CI: 0.25-0.70) and LSCC (HR: 0.29; 95\% CI: $0.25-0.70)$ (22). This discrepancy may be due to the use of serology for identifying HPV 16 E6 and E7, as opposed to examination of fresh-frozen or FFPE tumor specimens. Additionally, the tumor suppressor protein p16 has shown a similar prognostic role for OS in both nonOPSCC (HR: 0.41; 95\% CI: 0.25-0.69) and OPSCC (HR: 0.53; 95\% CI: 0.40-0.71) (16). A very recent study of HPVstatus in non-oropharyngeal tumors from the National Cancer Date Base (NCDB) found that HPV-status offered better OS for HPSCC and American Joint Committee on Cancer (AJCC) stage III to stage IVB LSCC and OCSCC (23). However, this large cohort study gathered data from 11 researches, which included different populations and used varied test method of high-risk HPV, and might cause some discrepancy from our findings.

Multivariate analysis and PSM identified HPV 16 status as an independent prognostic factor for survival in LSCC patients. These data are consistent with a previous study of 318 early stage LSCC cases, which showed that patients with $\mathrm{HPV}$-positive tumors had significantly improved OS $(\mathrm{P}=0.038)$ and RFS $(\mathrm{P}=0.006)$ compared to patients with HPV-negative tumors (24). Two additional studies found that HPV-positive LSCC patients had improved survival outcomes compared to HPV-negative patients, although these differences were not statistically significant $(25,26)$. In contrast, Young et al., found no statistically significant association between HPV RNA in situ hybridization status and OS in 80 patients with LSCC (27).

Survival outcomes based on HPV status in the oral cavity and hypopharynx vary between studies. Dahm et al., found that HPV status was not a significant factor in OS for patients with hypopharyngeal squamous cell carcinoma (HSCC) (HR: 0.53; 95\% CI: 0.14-1.35, $\mathrm{P}=0.175)$ (28). Lai et al., found no difference in OS $(\mathrm{P}=0.541)$ or diseasefree survival $(\mathrm{P}=0.723)$ between $\mathrm{HPV}$-positive and HPV- 
A

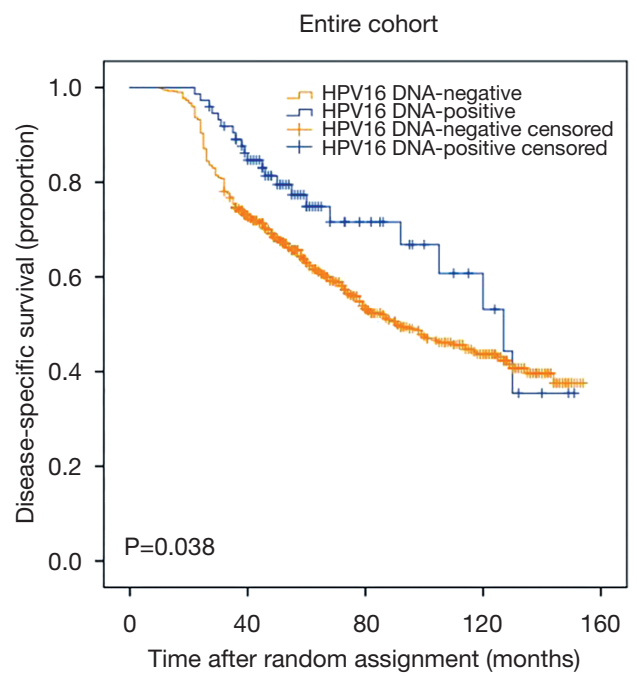

No. at risk

$\begin{array}{llllll}\text { HPV-positive } & 74 & 56 & 17 & 7 & 0\end{array}$

HPV-negative $\begin{array}{lllll}706 & 464 & 172 & 79 & 0\end{array}$

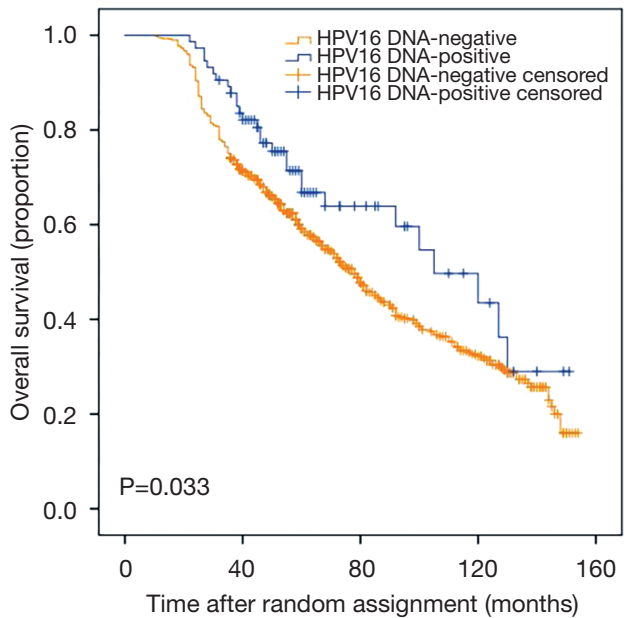

No. at risk

$\begin{array}{llllll}\text { HPV-positive } & 74 & 56 & 17 & 7 & 0\end{array}$

HPV-negative $\begin{array}{ccccc}706 & 464 & 172 & 77 & 0\end{array}$
B

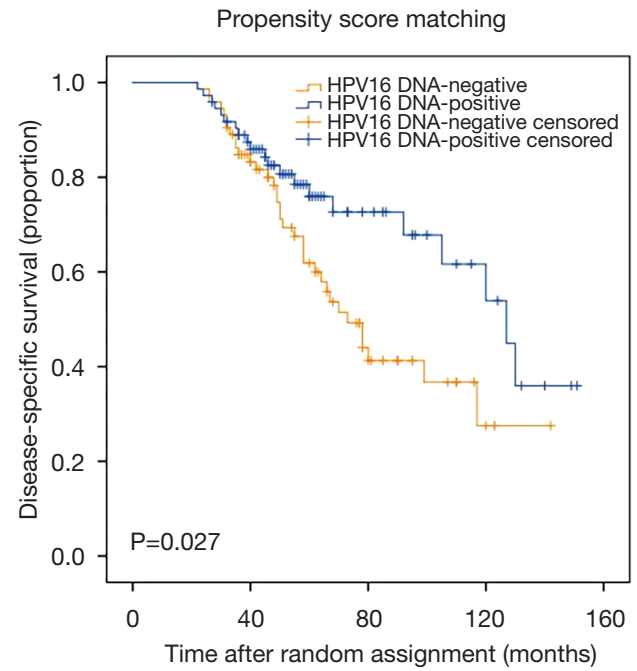

No. at risk

$\begin{array}{llllll}\text { HPV-positive } & 73 & 56 & 18 & 7 & 0\end{array}$

HPV-negative $\begin{array}{lllll}73 & 52 & 14 & 2 & 0\end{array}$

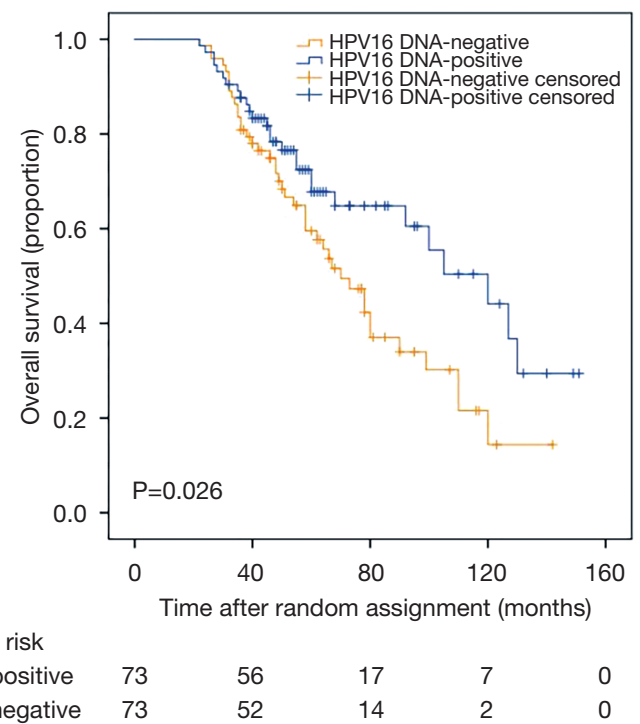

Figure 3 Disease-specific survival (DSS) and overall survival (OS) in HPV 16-positive and -negative LSCC patients before (A) and after (B) propensity score matching.

negative OCSCC patients (29). In contrast, a study based on data obtained from the National Cancer Database (NCDB) found that HPV-positive status was associated with improved survival outcomes for the oral cavity (HR: 0.76; 95\% CI: 0.66-0.87) and hypopharynx (HR: 0.59; 95\% CI: $0.45-0.77)(30)$. In the present study, no significant differences in OS or DSS were observed in either OCSCC or HPSCC patients, even after multivariate Cox regression and propensity score-adjusted analyses for bias reduction. A relatively small sample size and low HPV prevalence in OCSCC and HSCC patients may have introduced bias in our results. Further studies with larger cohorts containing a single tumor site are needed to verify these findings.

Several limitations of this study should be noted. First, this study was performed at a single institution, which may impact the generalization of our results. Second, other 
A

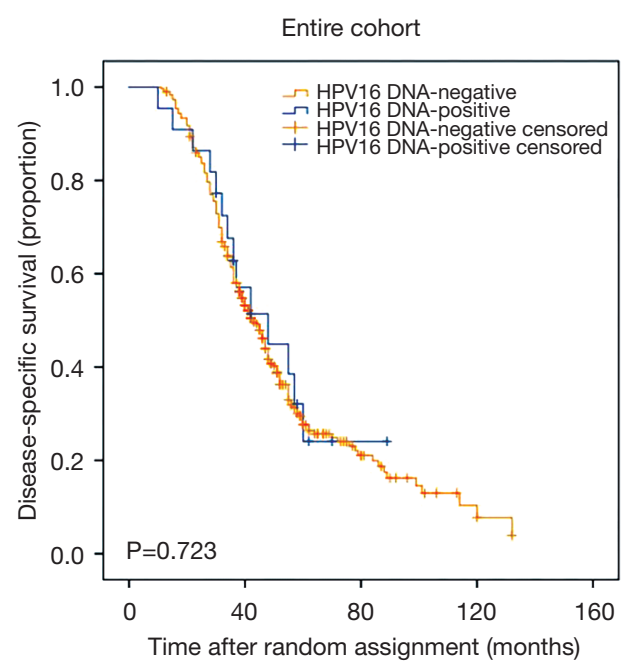

No. at risk

$\begin{array}{lcccll}\text { HPV-positive } & 22 & 9 & 1 & 0 & 0 \\ \text { HPV-negative } & 301 & 137 & 19 & 2 & 0\end{array}$

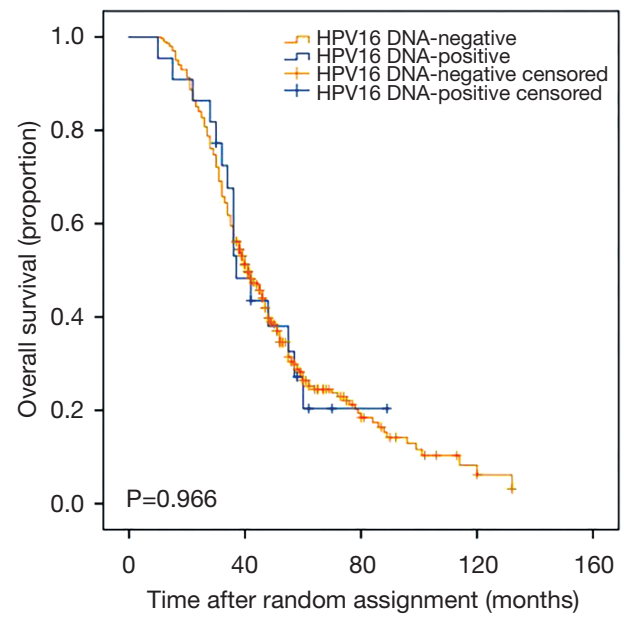

No. at risk

$\begin{array}{lccccc}\text { HPV-positive } & 22 & 9 & 1 & 0 & 0 \\ \text { HPV-negative } & 301 & 137 & 19 & 2 & 0\end{array}$

B

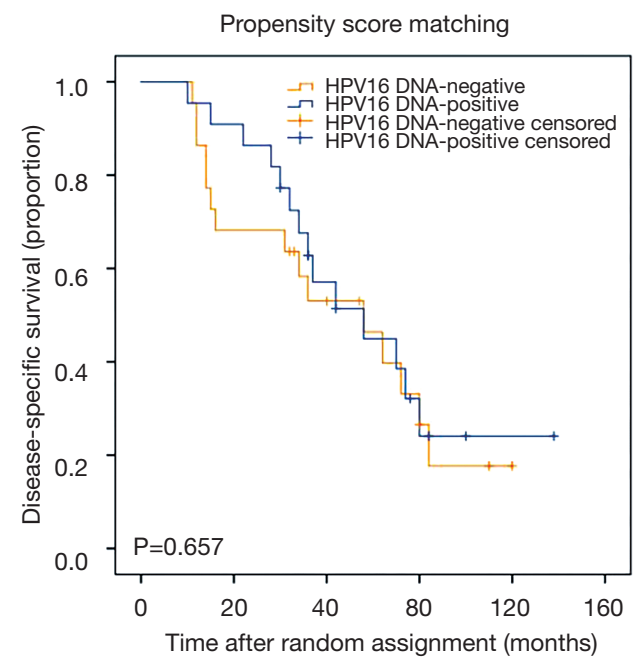

No. at risk

$\begin{array}{lllllll}\text { HPV-positive } & 22 & 19 & 9 & 3 & 1 & 0 \\ \text { HPV-negative } & 22 & 14 & 9 & 3 & 0 & 0\end{array}$

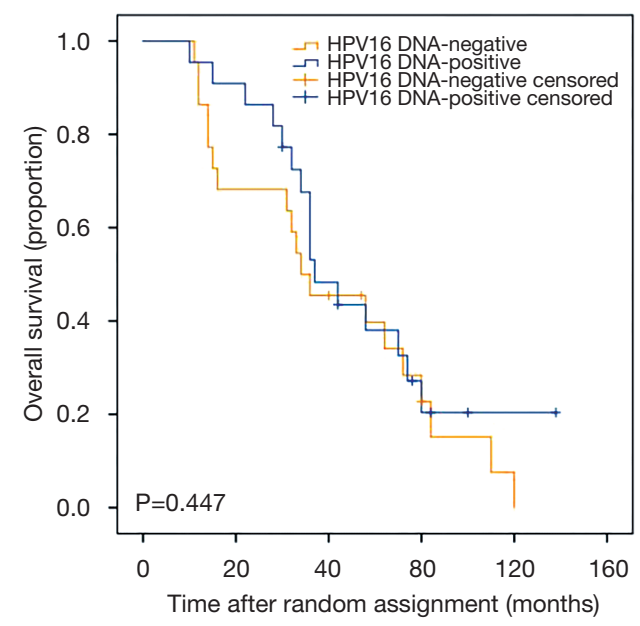

No. at risk

$\begin{array}{lllllll}\text { HPV-positive } & 22 & 19 & 9 & 3 & 1 & 0 \\ \text { HPV-negative } & 22 & 14 & 9 & 3 & 0 & 0\end{array}$

Figure 4 Disease-specific survival (DSS) and overall survival (OS) in HPV 16-positive and -negative HSCC patients before (A) and after (B) propensity score matching.

high-risk HPV types were not evaluated. Finally, HPV detection was not performed by E6/E7 mRNA qRT-PCR, which is the generally accepted standard for determining transcriptionally active HPV status (31). Because of these limitations, the relationship between HPV status and survival outcomes in non-OPSCC patients should be explored with prospective multi-institutional studies.

\section{Conclusions}

HPV 16-positive non-OPSCC patients do not have significantly better survival outcomes compared to HPV 16-negative patients. However, HPV 16-positive LSCC patients have significantly better survival outcomes compared to HPV 16-negative LSCC patients, which indicates that HPV 16 may be an independent prognostic factor for survival 
A

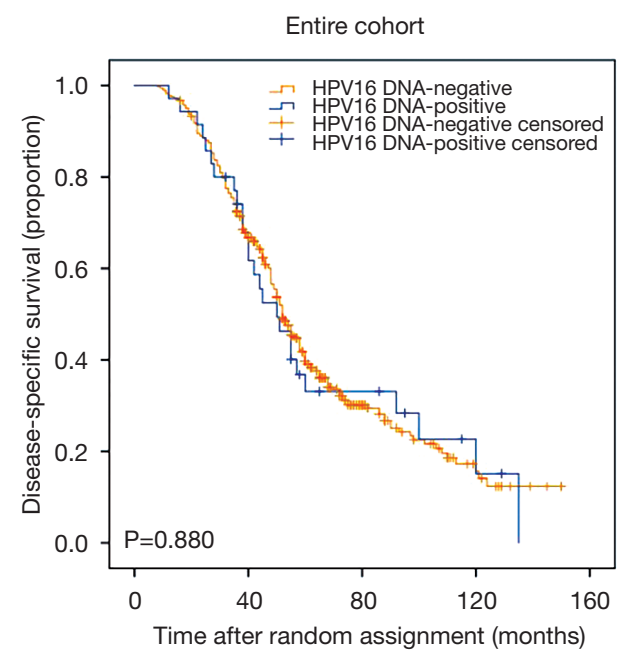

No. at risk

$\begin{array}{llllll}\text { HPV-positive } & 35 & 20 & 7 & 2 & 0\end{array}$

B

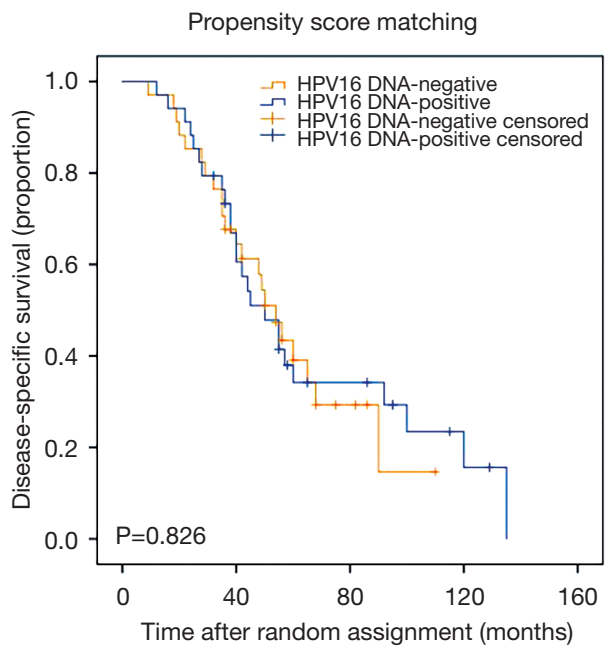

No. at risk

$\begin{array}{llllll}\text { HPV-positive } & 34 & 19 & 7 & 2 & 0 \\ \text { HPV-negative } & 34 & 20 & 3 & 0 & 0\end{array}$

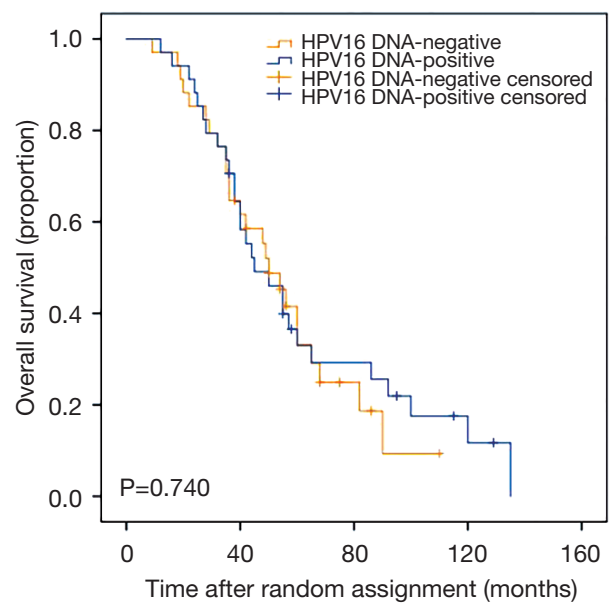

No. at risk

$\begin{array}{llllll}\text { HPV-positive } & 34 & 19 & 7 & 2 & 0 \\ \text { HPV-negative } & 34 & 20 & 3 & 0 & 0\end{array}$

No. at risk

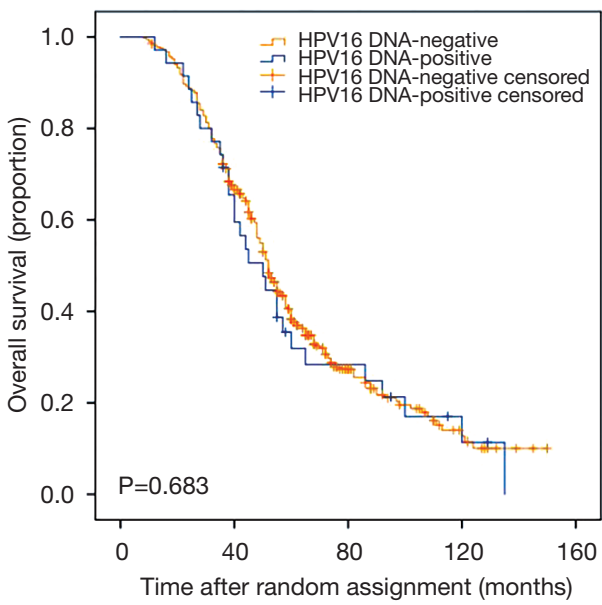

$\begin{array}{lccccc}\text { HPV-positive } & 35 & 20 & 7 & 2 & 0 \\ \text { HPV-negative } & 401 & 251 & 48 & 10 & 0\end{array}$

Figure 5 Disease-specific survival (DSS) and overall survival (OS) in HPV 16-positive and -negative OCSCC patients before (A) and after (B) propensity score matching.

in LSCC patients. Future studies with multicenter cohorts, larger sample sizes, and improved methods for defining HPV status are needed to verify these findings.

\section{Acknowledgments}

Funding: This work was supported by the National Natural Science Foundation of China (grant number 81273173).

\section{Footnote}

Conflicts of Interest: The authors have no conflicts of interest to declare.

Etbical Statement: The authors are accountable for all aspects of the work in ensuring that questions related to the accuracy or integrity of any part of the work are 
appropriately investigated and resolved. The Peking Union Medical College Hospital (PUMCH) Ethics Committee approved this study protocol. All participants provided informed written consent.

\section{References}

1. Bray F, Ferlay J, Soerjomataram I, et al. Global cancer statistics 2018: globocan estimates of incidence and mortality worldwide for 36 cancers in 185 countries. CA Cancer J Clin 2018;68:394-424.

2. Torre LA, Bray F, Siegel RL, et al. Global cancer statistics, 2012. CA Cancer J Clin 2015;65:87-108.

3. Maier H, Dietz A, Gewelke U, et al. Tobacco and alcohol and the risk of head and neck cancer. Clin Investig 1992;70:320-7.

4. Gillison ML, D'Souza G, Westra W, et al. Distinct risk factor profiles for human papillomavirus type 16-positive and human papillomavirus type 16-negative head and neck cancers. J Natl Cancer Inst 2008;100:407-20.

5. Tinhofer I, Johrens K, Keilholz U, et al. Contribution of human papilloma virus to the incidence of squamous cell carcinoma of the head and neck in a european population with high smoking prevalence. EUR J CANCER 2015;51:514-21.

6. Chaturvedi AK, Engels EA, Pfeiffer RM, et al. Human papillomavirus and rising oropharyngeal cancer incidence in the united states. J Clin Oncol 2011;29:4294-301.

7. Ang KK, Harris J, Wheeler R, et al. Human papillomavirus and survival of patients with oropharyngeal cancer. N Engl J Med 2010;363:24-35.

8. Rischin D, Young RJ, Fisher R, et al. Prognostic significance of p16INK4A and human papillomavirus in patients with oropharyngeal cancer treated on TROG 02.02 phase III trial. J Clin Oncol 2010;28:4142-8.

9. Castellsagué X, Alemany L, Quer M, et al. HPV Involvement in Head and Neck Cancers: Comprehensive Assessment of Biomarkers in 3680 Patients. J Natl Cancer Inst 2016;108:djv403.

10. Mehanna H, Beech T, Nicholson T, et al. Prevalence of human papillomavirus in oropharyngeal and nonoropharyngeal head and neck cancer-systematic review and meta-analysis of trends by time and region. Head Neck 2013;35:747-55.

11. Chen X, Gao L, Sturgis EM, et al. HPV16 DNA and integration in normal and malignant epithelium: implications for the etiology of laryngeal squamous cell carcinoma. Ann Oncol 2017;28:1105-10.

12. Ndiaye C, Mena M, Alemany L. Correction to lancet oncol 2014; 15: 1324. HPV DNA, E6/E7 mRNA, and p16INK4A detection in head and neck cancers: a systematic review and meta-analysis. Lancet Oncol 2015;16:e262.

13. Ragin CC, Taioli E. Survival of squamous cell carcinoma of the head and neck in relation to human papillomavirus infection: review and meta-analysis. Int J Cancer 2007;121:1813-20.

14. Isayeva T, Li Y, Maswahu D et al. Human papillomavirus in non-oropharyngeal head and neck cancers: a systematic literature review. Head Neck Pathol 2012;6 Suppl 1:S104-20.

15. Chung $\mathrm{CH}$, Zhang Q, Kong CS, et al. P16 protein expression and human papillomavirus status as prognostic biomarkers of nonoropharyngeal head and neck squamous cell carcinoma. J Clin Oncol 2014;32:3930-8.

16. Bryant AK, Sojourner EJ, Vitzthum LK, et al. Prognostic role of p16 in nonoropharyngeal head and neck cancer. J Natl Cancer Inst 2018;110:1393-9.

17. Nesic VS, Petrovic ZM, Sipetic SB, et al. Comparison of the adult comorbidity evaluation 27 and the charlson comorbidity indices in patients with laryngeal squamous cell carcinoma. J Laryngol Otol 2012;126:516-24.

18. Anaya-Saavedra G, Ramirez-Amador V, Irigoyen-Camacho ME, et al. High association of human papillomavirus infection with oral cancer: a case-control study. Arch Med Res 2008;39:189-97.

19. Elze MC, Gregson J, Baber U, et al. Comparison of propensity score methods and covariate adjustment: evaluation in 4 cardiovascular studies. J Am Coll Cardiol 2017;69:345-57.

20. Martens EP, Pestman WR, de Boer A, et al. Systematic differences in treatment effect estimates between propensity score methods and logistic regression. Int J Epidemiol 2008;37:1142-7.

21. Fakhry C, Westra WH, Wang SJ, et al. The prognostic role of sex, race, and human papillomavirus in oropharyngeal and nonoropharyngeal head and neck squamous cell cancer. Cancer 2017;123:1566-75.

22. Nelson HH, Pawlita M, Michaud DS, et al. Immune Response to HPV16 E6 and E7 Proteins and Patient Outcomes in Head and Neck Cancer. JAMA Oncol 2017;3:178-85.

23. Tian S, Switchenko JM, Jhaveri J, et al. Survival outcomes by high-risk human papillomavirus status 
in nonoropharyngeal head and neck squamous cell carcinomas: A propensity-scored analysis of the National Cancer Data Base. Cancer 2019;125:2782-93.

24. Wang H, Zhang Z, Sun R, et al. HPV infection and anemia status stratify the survival of early T2 laryngeal squamous cell carcinoma. J Voice 2015;29:356-62.

25. Stephen JK, Chen KM, Shah V, et al. Human papillomavirus outcomes in an access-to-care laryngeal cancer cohort. Otolaryngol Head Neck Surg 2012;146:730-8.

26. $\mathrm{Xu} \mathrm{Y,} \mathrm{Liu} \mathrm{S,} \mathrm{Yi} \mathrm{H,} \mathrm{et} \mathrm{al.} \mathrm{Human} \mathrm{papillomavirus} \mathrm{infection}$ in 674 chinese patients with laryngeal squamous cell carcinoma. PLoS One 2014;9:e115914.

27. Young RJ, Urban D, Angel C, et al. Frequency and prognostic significance of p16(INK4A) protein overexpression and transcriptionally active human papillomavirus infection in laryngeal squamous cell

Cite this article as: Zhu Y, Xia X, Gao L, Zhu X, Diao W, Liang Z, Gao Z, Chen X. Prognostic implications of human papillomavirus type 16 status in non-oropharyngeal head and neck cancer: a propensity score matching analysis. Ann Transl Med 2019;7(23):759. doi: 10.21037/atm.2019.11.72 carcinoma. Br J Cancer 2015;112:1098-104.

28. Dahm V, Haitel A, Kaider A, et al. Cancer stage and packyears, but not p16 or hpv, are relevant for survival in hypopharyngeal and laryngeal squamous cell carcinomas. Eur Arch Otorhinolaryngol 2018;275:1837-43.

29. Lai K, Killingsworth M, Matthews S, et al. Differences in survival outcome between oropharyngeal and oral cavity squamous cell carcinoma in relation to HPV status. J Oral Pathol Med 2017;46:574-82.

30. Li H, Torabi SJ, Yarbrough WG, et al. Association of human papillomavirus status at head and neck carcinoma subsites with overall survival. JAMA Otolaryngol Head Neck Surg 2018;144:519-25.

31. Shi W, Kato H, Perez-Ordonez B, et al. Comparative prognostic value of HPV16 E6 mRNA compared with in situ hybridization for human oropharyngeal squamous carcinoma. J Clin Oncol 2009;27:6213-21. 